\title{
Historia natural de la lesión de plexo braquial de nacimiento
}

\author{
Natural history of brachial plexus birth injury \\ Leonardo López Almejo, ${ }^{*}$ José Armando Romero Rocha, ${ }^{\ddagger}$ \\ Luis Carlos Urzúa Portillo, ${ }^{\ddagger}$ Francisco Yamil Pérez Lima, ${ }^{\ddagger}$
}

Ana Fernanda Espinosa de los Monteros Kelley, ${ }^{\S}$ René Humberto Barraza Arrambide, ${ }^{\S}$ Pablo Zancolli, ${ }^{\S}$ Raúl Emérico Rodríguez Martínez, ${ }^{\S}$ Rogelio Josúe Solano Pérez,"

Nemesio Hernández Rodríguez," José Eduardo Magaña Chávez, ** Israel Gutiérrez Mendoza, ${ }^{\ddagger}$ Jorge Francisco Clifton Correa ${ }^{\S \S}$

\footnotetext{
* Vicepresidente FEMECOT, Coordinador del Comité Científico FEMECOT. Cirujano Ortopedista

UNAM, Cirujano de Mano UAG, Profesor Titular del Postgrado en Cirugía de Plexo Braquial y Nervio periférico. Universidad Autónoma de Coahuila. Máster en Cirugía de Plexo Braquial, Brachial Plexus International School (BPIS). Clínica Mielina Aguascalientes.

₹ Cirujano Ortopedista, Diplomado en Cirugía de Plexo Braquial. Hospital Universitario. Universidad Autónoma de Coahuila.

$\S$ Cirujano Ortopedista. Master en Cirugía de Plexo Braquial y Nervio Periférico. BPIS. Cirujano de Mano.

" Cirujano Ortopedista. Master en Cirugía de Plexo Braquial y Nervio Periférico BPIS. Cirujano de Columna.

॥ Cirujano Ortopedista, Profesor de Cirugía de Plexo Braquial. Hospital Universitario. Universidad Autónoma de Coahuila.

${ }^{* \star}$ Cirujano Ortopedista. Clínica Mielina Aguascalientes. Hospital Santo Toribio Romo, Villa Hidalgo, Jal.

\# Cirujano Ortopedista. Maestría en Ciencias Médicas. Profesor Universidad Cuauhtémoc. Clínica Mielina Aguascalientes.

\$s Cirujano Plástico. Profesor de Cirugía de Plexo Braquial y Nervio Periférico. Hospital Universitario.

Universidad Autónoma de Coahuila. Profesor del Máster en Cirugía de Plexo Braquial BPIS.
}

Correspondencia: Leonardo López Almejo. Hospital Star Médica. Av. Universidad 103, Consultorio 219, Col. Villas de la Universidad, 20020, Aguascalientes, Ags. México, Tel: 44-9413-9901.

Correo electrónico: lyon77777@gmail.com

ORCID ID: 0000-0002-6104-2349

\section{RESUMEN}

La lesión del plexo braquial de nacimiento es una parálisis de las extremidades superiores que se produce debido a una lesión por tracción del plexo braquial durante el parto. Se define como una paresia flácida de un brazo al nacer con un rango de movimiento pasivo mayor que el activo. Aproximadamente $20 \%$ de los niños con parálisis del plexo braquial al

\section{ABSTRACT}

Brachial plexus birth palsy is an upper extremity paralysis that occurs due to traction injury of the brachial plexus during childbirth. Is defined as a flaccid paresis of an arm at birth with a passive range of motion greater than the active. Approximately 20\% of children with brachial plexus birth palsy will have residual neurologic deficits. The

Citar como: López AL, Romero RJA, Urzúa PLC, Pérez LFY, Espinosa de los Monteros KAF, Barraza ARH y cols. Historia natural de la lesión de plexo braquial de nacimiento. Ortho-tips. 2020; 16 (4): 162-172. https://dx.doi.org/10.35366/97815 
nacer tendrán déficits neurológicos residuales. Se diagnostica debido a la falta de movimiento espontáneo de la extremidad superior. El examen clínico también puede revelar una pérdida del movimiento de los reflejos, además de la presencia del síndrome de Horner.

Palabras clave: Lesión del plexo braquial, parálisis del plexo braquial congénito, lesión perinatal, parálisis superior de la extremidad, displasia glenohumeral, lesión obstétrica del plexo braquial. diagnosis is made by the lack of spontaneous movement of the upper limb. The clinical examination may also reveal a loss of reflex movement, in addition to the presence of Horner's sign.

Keywords: Brachial plexus injury, brachial plexus birth palsy, perinatal injury, upper palsy extremity, glenohumeral dysplasia, obstetrical brachial plexus injury.

\section{INTRODUCCIÓN}

La lesión del plexo braquial de nacimiento (LPBN) es una parálisis de la extremidad superior que se produce debido a una tracción del plexo braquial durante el trabajo de parto. En la mayoría de los casos, la sínfisis púbica de la madre bloquea el parto en la parte superior del hombro (distocia del hombro). Si se aplica tracción adicional a la cabeza del niño, el ángulo entre el cuello y el hombro se aumenta con fuerza, estirando demasiado el plexo braquial ipsilateral. La lesión por tracción resultante puede variar en la severidad del daño durante el parto.

Se define como una paresia flácida de un brazo al nacer con un rango de movimiento pasivo mayor que el activo. Se diagnostica típicamente debido a la falta de movimiento espontáneo de las extremidades superiores. El examen clínico también puede revelar una pérdida del movimiento de los reflejos así como la presencia del síndrome de Horner (Figura 1). La LPBN es una de las lesiones más frecuentes del recién nacido. Hasta ahora, la extensión del daño neural sólo puede evaluarse observando la recuperación en el transcurso del tiempo porque las lesiones nerviosas de diferente gravedad se presentan inicialmente con las mismas características clínicas. ${ }^{1-3}$

\section{CONCEPTOS ANATÓMICOS}

El plexo braquial está conformado por los nervios cervicales de C5 a C8 y por el pri-

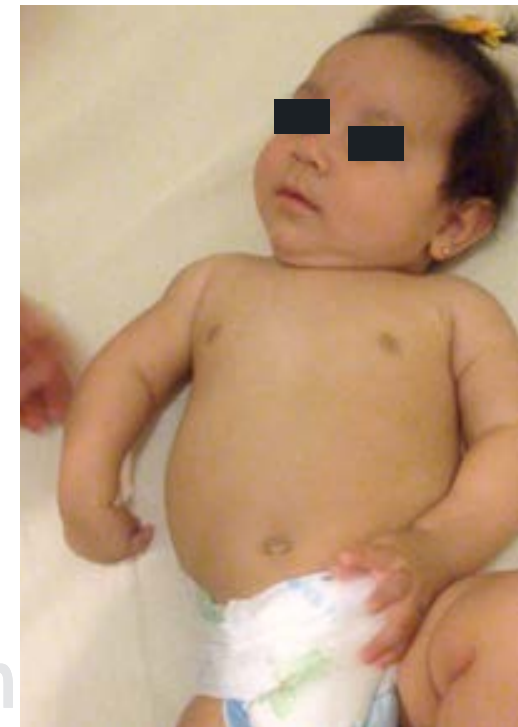

Figura 1: Paciente femenino de nueve meses con lesión del plexo braquial de nacimiento con síndrome de Horner. Imagen del autor. 

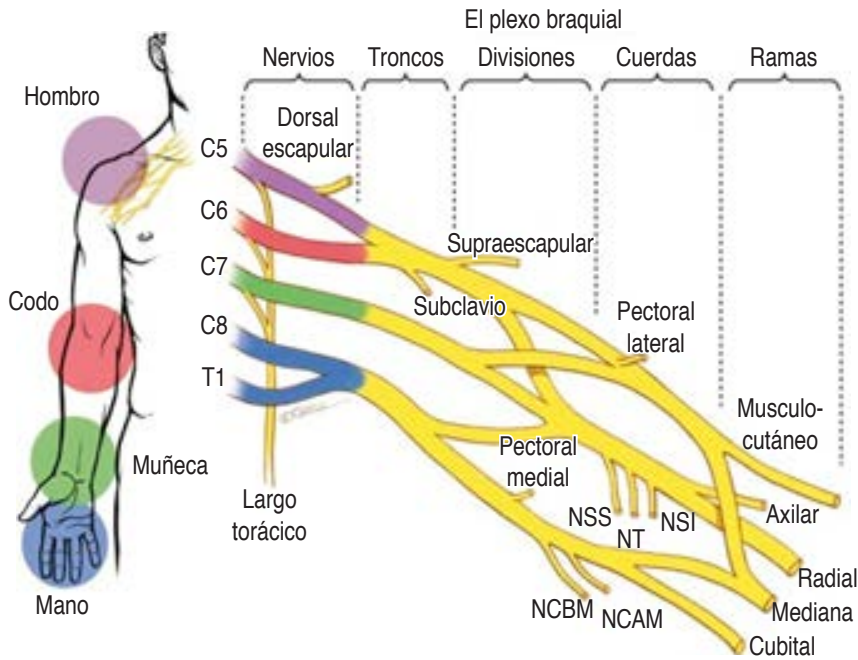

Figura 2:

Anatomía del plexo braquial. La localización de las lesiones del plexo braquial depende de la comprensión detallada de su anatomía. Esta imagen la utilizamos al momento de la exploración de cada paciente para determinar el nivel de lesión. Modificado de la Fundación Mayo para la Educación e Investigación Médica. Todos los derechos reservados. $\mathrm{NSI}=$ nervio subescapular inferior; NCAM = nervio cutáneo antebraquial medial; NCBM = nervio cutáneo braquial medial; $\mathrm{NT}=$ nervio toracodorsal; NSS $=$ nervio subescapular superior.

mer nervio torácico (T1). Sus raíces nerviosas son responsables de la inervación sensitiva y motora de las extremidades superiores hasta en $75 \%$ de la población. En algunas ocasiones puede recibir una contribución del cuarto nervio cervical (C4 hasta en $22 \%$ de la población), lo que se conoce como plexo prefijado, o del segundo nervio torácico (T2 hasta en 1-3\% de la población), llamado plexo postfijado. Cuando el plexo es prefijado tiene una disposición en el cuello más vertical, y cuando es postfijado, más horizontal. La ubicación topográfica lo hace más susceptible a traumatismos debido a su proximidad anatómica a las estructuras oscilantes del cuello y los hombros, y a la ausencia de protección muscular y ósea (Figura 2).

El plexo braquial pasa entre los músculos escaleno anterior y medio con sus troncos de ubicación supraclavicular y los cordones en posición infraclavicular. El tronco primario superior del plexo braquial suele estar formado por los nervios $\mathrm{C} 5$ y C6, el tronco medio está formado por el $\mathrm{C} 7$, y el tronco inferior por C8 y T1. ${ }^{4-8}$ Posteriormente, cada tronco generará una división posterior y una anterior. La combinación de éstas dará origen a los cordones lateral, medial y posterior y finalmente de estos cordones se formarán las ramas terminales principales como el nervio musculocutáneo, axilar, radial, mediano y cubital.

\section{INCIDENCIA}

La LPBN es una lesión común y de impacto en la salud pública. Tanto la incidencia como el pronóstico de estas lesiones son inciertos. La incidencia notificada varía 10 veces, de 0.4 por 1,000 a 4.6 por 1,000 y la proporción con la lesión varía de 12 a $50 \%$. Otras referencias informan que son afectados de uno a cuatro niños por cada 1,000 nacidos vivos. Esta incidencia sigue en aumento debido al mayor peso al nacer, a pesar de los avances en la atención obstétrica. ${ }^{8,9}$ La prevalencia de esta lesión en los Estados Unidos disminuyó de 1.7 a 0.9 por 1,000 nacidos 

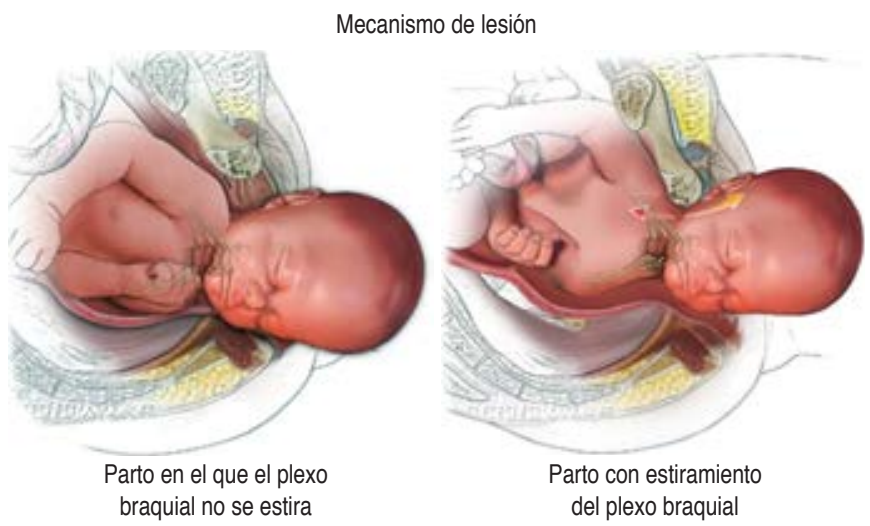

Figura 3:

Imagen que ejemplifica la tracción cefálica, un riesgo de lesión del plexo braquial de nacimiento. Modificado de: www.vitalclinic. es/paralisis-braquial-obstetricia.

vivos de 1997 a 2012 en el contexto de tasas decrecientes de macrosomía y tasas crecientes de multiparidad y cesárea. Las variaciones en la tasa informada pueden atribuirse a diferencias geográficas en la privación socioeconómica, la atención obstétrica y el peso al nacer. ${ }^{10}$

\section{FACTORES DE RIESGO}

El plexo braquial es vulnerable tanto en el periodo perinatal como en el postnatal. Comprender las variaciones morfológicas del plexo braquial es fundamental en neurología y traumatología, y estas alteraciones pueden explicar signos y síntomas clínicos inesperados, por lo que algunos estudios muestran la importancia de estudiar el plexo braquial en fetos humanos. ${ }^{6,11}$

En los recién nacidos, la mayoría de las parálisis del plexo braquial se produce debido al alargamiento del plexo braquial durante el parto al separar la cabeza con fuerza del hombro (Figura 3); sin embargo, existen casos documentados de esta lesión sin tracción de la cabeza fetal, y varios autores se han opuesto al término «parálisis obstétrica» debido, entre otros factores, a los avances en la obstetricia moderna.

Existen otros factores de riesgo asociados que podemos dividir en:

\section{Factores de riesgo maternos}

- Anomalías uterinas. La presión prenatal de larga duración sobre el plexo bra-

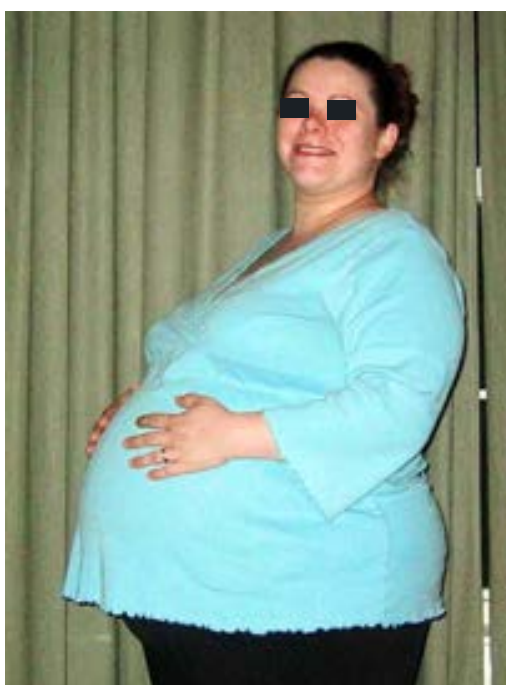

Figura 4: Imagen de una mujer embarazada con obesidad. Tomado de: www.elmundous.com 
quial se produce con anomalías uterinas como los miomas uterinos, un útero bicornado o una posición transversal prolongada).

- Estatura materna baja.

- Diabetes mellitus (DM) pregestional o gestacional. Principalmente la DM insulinodependiente (los bebés de madres diabéticas tienen mayor incidencia de lesión relacionada con las características del tipo de cuerpo fetal y la macrosomía) en comparación con los lactantes macrosómicos, cuya madre era sana (sin diabetes).

- Obesidad (Figura 4).

\section{Factores de riesgo infantil}

- Macrosomía. Se define como el peso al nacer de 4,500 g. Hay una incidencia de 45 a 100 veces más de presentar una LPBN (Figura 5). El índice de grasa corporal total es mayor y el diámetro de hombros y tórax son más grandes, lo que dificulta el parto y propicia las lesiones.

- Variaciones anatómicas y compromiso neurológico.

- Anomalías esqueléticas infantiles como la presencia de una costilla cervical.

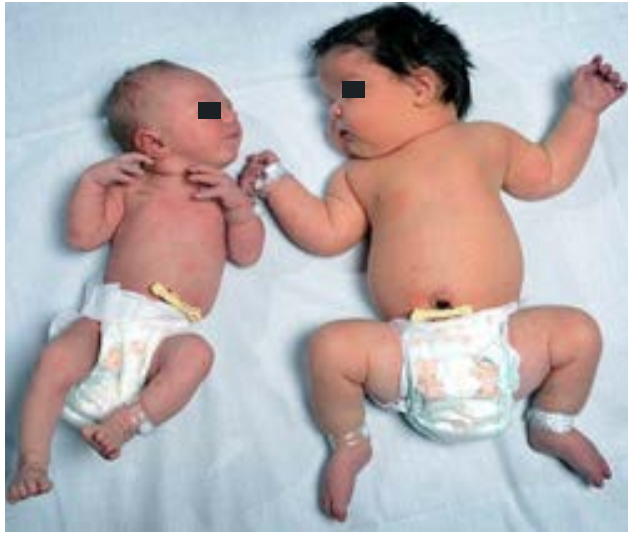

Figura 5: Imagen de dos recién nacidos con peso distinto entre ellos. El de la izquierda con un peso de $2,857 \mathrm{~g}$ al nacer y el de la derecha con $5,443 \mathrm{~g}$ Tomado de: www.dailytelegraph.com.au.

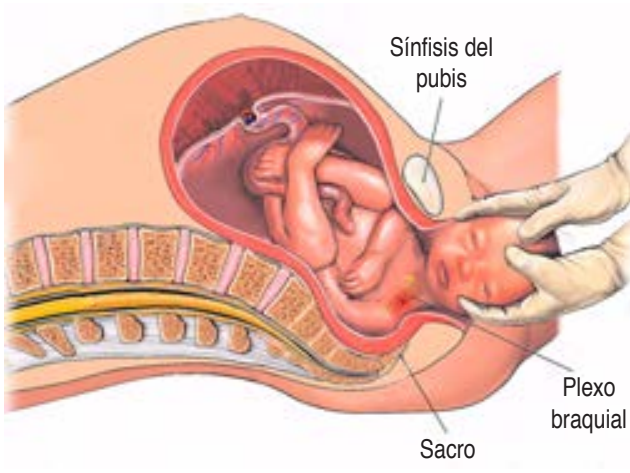

Figura 6: Imagen que muestra el aumento del ángulo cabeza-cuello.

Modificado de: https://www.choa.org/medicalservices/orthopaedics/brachial-plexus.

- Las infecciones como la varicela congénita pueden causar lesión isquémica del plexo braquial en el útero, haciéndolo menos tolerante a la tracción.

\section{Factores de riesgo asociados al parto}

- La distocia de hombros. Proceso en el que el hombro del feto queda atrapado contra la sínfisis del pubis materno, creando tensión en la parte superior del plexo braquial fetal. También se ha definido como el retraso de 60 segundos en el intervalo entre el parto de la cabeza y el parto del cuerpo, junto con el uso de maniobras obstétricas más allá de la aplicación de técnicas de tracción 
suave para liberar el hombro obstruido. Dada por la discrepancia entre el tamaño y/o la orientación del hombro fetal en el ingreso a la pelvis materna que impacta detrás de la sínfisis del pubis o del promontorio del sacro, produciendo compresión y estiramiento del plexo braquial. Posterior a esto se generan las fuerzas mecánicas de tracción. Dentro de ellas la tracción lateral producida puede aumentar el ángulo entre la cabeza y el hombro (Figura 6), transfiriendo la fuerza que puede elongar o romper aún más el plexo braquial, generando una variedad de lesiones que pueden ir de leves a una forma severa de limitación.

- Partos pélvicos con hiperextensión cervical del recién nacido y la obesidad (Figura 7).

- Parto prolongado. Las fuertes contracciones uterinas y las fuerzas maternas de empuje pueden comprimir y elongar el plexo braquial, no necesariamente dadas por la fuerza generada por el operador del parto. Estudios de ingeniería matemática y biomédica demuestran que las fuerzas endógenas (contracciones uterinas-esfuerzos maternos de empuje) pueden generar lesión en el plexo braquial.

- Parto previo con antecedente de LPBN.

- La asfixia perinatal. Ésta provoca hipotonía, lo que predispone a las lesiones del plexo por estiramiento. Se ha observado una relación entre la puntuación de Apgar de $<5$ a los cinco minutos y la LPBN.

- Parto instrumentado (uso de fórceps) aumenta el riesgo de LPBN hasta nueve veces más.

- Taquisítole. Se define como la presencia de más de seis contracciones uterinas en 10 minutos, o una gran contracción (sostenida) que se mantiene por más de dos minutos, aumentando el riesgo de LPBN hasta de 3.7 más.

- Uso de oxitocina. Puede generar contracción continua irregular, puede ocasionar riesgo de 2.5 veces más de presentar una LPBN.

- Posición de la madre al momento del parto. Se ha revisado en diferentes modelos que la posición de McRoberts (hiperflexión de las caderas maternas) reduce las fuerzas de entrega por el operador y creadas por la madre sobre el plexo braquial.

- Posición del brazo en el nacimiento. Se ha observado mayor relación en la afectación del brazo derecho $0^{6,8,12-19}$

\section{Factores de protección}

- Peso menor de 3,000 g

- Parto gemelar o múltiple

- Cesárea (disminuye el riesgo, pero no lo elimina por completo)

Tipo de lesión. Los tipos de lesión que se pueden presentar son:

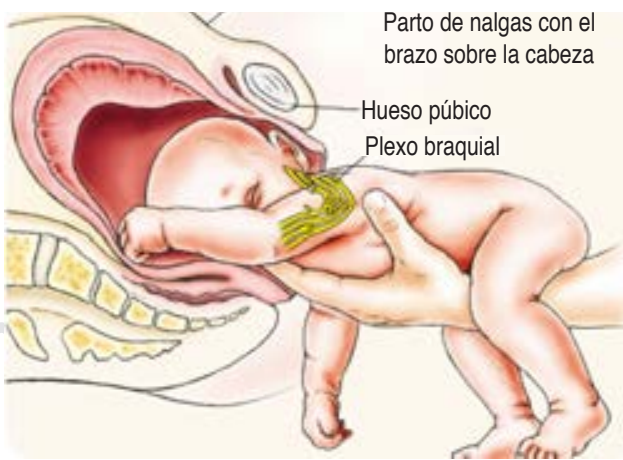

Figura 7: Imagen que ejemplifica un parto pélvico. Modificado de: https://www.seattlechildrens.org/pdf/PE1025.pdf. 


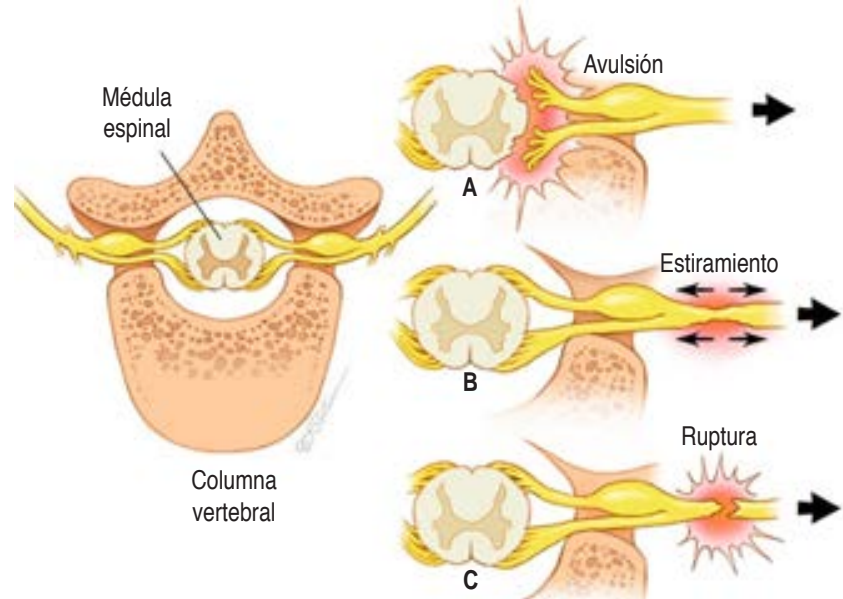

${ }^{\odot}$ Fundación Mayo para la Educación e Investigación Médica, todos los derechos reservados.
Figura 8:

Esquema donde se indica los tipos de lesión. Avulsión, elongación y ruptura. Modificado de: https://www. mayoclinic.org/es-es/diseasesconditions/brachial-plexus-injury/ symptoms-causes/syc-20350235.

- Elongación

- Ruptura

- Avulsión

El tipo de lesión nerviosa más grave es la avulsión (A), que se produce cuando las raíces nerviosas se desprenden de la médula espinal. Las lesiones menos graves consisten en un estiramiento (B) de las fibras nerviosas o una ruptura $(C)$, que se produce cuando el nervio se rompe en partes separadas (Figura 8).

\section{DIAGNÓSTICO}

Los pacientes con LPBN generalmente se diagnostican después del nacimiento debido a la falta de movimiento en el hombro, el codo, la muñeca y/o los dedos. Para ello es necesario obtener un historial de nacimiento completo para evaluar los factores de riesgo así como para conocer los puntajes Apgar porque los niños con parálisis del plexo braquial pueden haber experimentado anoxia cerebral durante el proceso de parto. La estimulación táctil se utiliza en un intento de alentar al bebé a mover la extremidad. Además, se pueden evaluar los reflejos neonatales (ejemplo reflejo de Moro). La evaluación del síndrome de Horner también se realiza buscando ptosis, miosis y/o anhidrosis, que de estar presentes implica un mal pronóstico para el bebé. El examen físico es la herramienta principal de evaluación al revisar a un recién nacido. Con un examen cuidadoso de grupos de músculos específicos, el médico puede determinar el patrón de afectación de la raíz nerviosa. También debe evaluar, a través de la palpación, una fractura de clavícula o húmero, que puede coexistir con una parálisis de nacimiento del plexo braquial o causar una pseudoparálisis, ya que el niño resiste el movimiento secundario al dolor. La presencia de espasticidad durante el examen puede indicar 
que el bebé tiene un período de anoxia cerebral. Una vez que se establece un diagnóstico, se necesitan exámenes seriados para predecir la recuperación y sí se necesitan intervenciones adicionales. La utilización de estudios radiológicos o de electrodiagnóstico adicional es algo controvertida porque un buen examen físico proporciona la información necesaria para planear el manejo y ninguna de las modalidades puede predecir uniformemente el patrón exacto de lesión nerviosa. 8,10,20,21 Existen escalas de apoyo diagnóstico que nos ayudan a evaluar las lesiones. Una de las más completas y que en nuestro grupo utilizamos, es la escala de movimiento activo del Hospital para el Niño Enfermo de Toronto, ${ }^{21,22}$ que evalúa el plexo braquial utilizando 15 movimientos diferentes de la extremidad superior, emplea una puntuación de 0 a 7 y evalúa cada movimiento sin gravedad y luego contra la gravedad, intentando pronosticar la recuperación neurológica con una puntuación compuesta.

La puntuación de Mallet modificada ${ }^{23,24}$ es más útil para los niños pequeños y mayores, y se basa en medir movimientos funcionales generales como la abducción del hombro, la rotación externa del hombro y otros movimientos, incluidos el llevarse la mano a la boca, al cuello, a la columna y al abdomen.

En conclusión, el diagnóstico se basa en la exploración física a través del movimiento pasivo y doloroso del miembro afectado, ausencia de movimiento activo, parálisis flácida, pérdida del patrón flexor y cambios tróficos de la piel, y nos genera un factor de mal pronóstico la presencia del síndrome de Horner. El factor más favorable es la presencia temprana de la flexión del codo contra gravedad, ya que el retorno tardío de la función del bíceps se ha asociado con puntuaciones inferiores y pronóstico no favorable. . $1,22,25-28$

\section{CLASIFICACIÓN}

La LPBN se clasifica según las raíces nerviosas afectadas. La parálisis de Erb clásica es una lesión que involucra las raíces nerviosas C5 y C6 y es el patrón de lesión más común, y se presenta en aproximadamente $60 \%$ de los casos. Una parálisis de Erb extendida afecta las raíces nerviosas C5, C6 y C7 y representa aproximadamente de 20 a $30 \%$ de los casos. Cuando todas las raíces nerviosas están dañadas (C5 a T1), esto se denomina parálisis total del plexo braquial y representa de 15 a $20 \%$ de los casos. Las lesiones aisladas de las raíces nerviosas C8 y T1, denominadas parálisis de Klumpke, son extremadamente raras y representan menos de $1 \%$ de los casos.

Existen diferentes tipos de lesiones nerviosas para cada nivel de la raíz afectada que incluyen neuropraxia, axontomesis, neurotmesis y avulsión. La neuropraxia representa la pérdida de mielina y se debe obtener una recuperación completa en varias semanas. Por el contrario, las lesiones por neurotomesis y avulsión requieren procedimientos microquirúrgicos tempranamente para restaurar la función, pero tienen un mal pronóstico. Las lesiones por axonotomesis pueden tener una recuperación funcional completa, una recuperación parcial o ningún tipo de recuperación, dependiendo de la extensión del daño axonal presentado y el número de raíces involucradas. ${ }^{8,14,29,30}$ 


\section{PRONÓSTICO}

El pronóstico dependerá de los factores de riesgo presentados, el tipo de lesión, la extensión del daño y la fecha de inicio del tratamiento médico y/o quirúrgico. Aun cuando hay estudios multicéntricos recientes que indican que no modifica el pronóstico realizar una cirugía temprana (edad promedio 4.2 meses) en comparación con la cirugía tardía (edad promedio 10.7 meses), ${ }^{31}$ no se encontraron diferencias funcionales con seguimiento a uno o dos años en un grupo de 32 pacientes, los cuales fueron tratados con injertos nerviosos o transferencias nerviosas. ${ }^{32}$ Con base en una revisión sistemática que dependerá de la raíz afectada, los datos internacionales combinados favorecen fuertemente la transferencia nerviosa dual sobre el injerto de nervio tradicional en lesiones C5-C6. Más aún cuando se contemplan injertos de nervios largos. ${ }^{33}$

\section{RECOMENDACIONES}

Con base en la fisiopatología de la lesión y conocimientos de la regeneración nerviosa es fundamental el diagnóstico temprano, el manejo médico-quirúrgico adecuado (rehabilitación, reconstrucción nerviosa, transferencias nerviosas, transferencias tendinosas) y el seguimiento y tratamiento de las secuelas.

1. Se debe examinar físicamente al recién nacidos para detectar LPBN.

2. Derivar a todos los recién nacidos con lesión o sospecha de LPBN a un centro multidisciplinario con experiencia en el manejo de estas lesiones antes del mes de edad. El consenso de expertos dice que es «bueno» si se envía al mes de edad, «satisfactorio» si se envía a los tres meses y «malo» posterior a los tres meses.

3. Proporcione antecedentes completos del embarazo y nacimiento, y los hallazgos del examen físico (incluido el síndrome de Horner) al nacer.

4. Los centros multidisciplinarios deben incluir: un médico rehabilitador con experiencia en el manejo de estas lesiones, y un cirujano de nervios periféricos con experiencia en reparación microquirúrgica. ${ }^{34}$

5. El tratamiento no quirúrgico fuera de un centro multidisciplinario debe ser asesorado por un equipo especializado.

6. El manejo microquirúrgico del nervio debe emplearse para todas las lesiones que cumplan con los criterios operativos definidos por el centro multidisciplinario a partir de los tres meses de edad.

7. Para la recopilación objetiva de resultados se debe clasificar la lesión (Narakas ${ }^{35,36}$ desde la valoración inicial, la longitud de la extremidad, la escala de movimiento activo (AMS) y la evaluación de los resultados a uno, tres, seis, 12 y 24 meses de edad, después dar seguimiento anualmente.

Al día de hoy, los instrumentos de medición de la LPBN se centran principalmente en la capacidad física de las actividades de la vida diaria y el bienestar psicosocial del niño. Una atención temprana mejora la conciencia de sus extre- 
midades y ayuda a los terapeutas y a los padres a personalizar el tratamiento de una manera más centrada en el manejo de las secuelas. ${ }^{37}$

Las recomendaciones de derivación son más efectivas si los especialistas locales están involucrados en la difusión y hay formularios de derivación estructurados. Para mejorar la derivación hay que generar información. Solo un grupo multidisciplinario puede conectar la educación y la conciencia pública, la modificación de los factores de riesgo, la derivación, la evaluación y la terapia. ${ }^{34,38}$

\section{BIBLIOGRAFÍA}

1. Buterbaugh $\mathrm{KL}$, Shah AS. The natural history and management of brachial plexus birth palsy. Curr Rev Musculoskelet Med. 2016; 9 (4): 418-426. doi: 10.1007/s12178-016-9374-3.

2. Evans-Jones G, Kay SP, Weindling AM, et al. Congenital brachial palsy: incidence, causes, and outcome in the United Kingdom and Republic of Ireland. Arch Dis Child Fetal Neonatal Ed. 2003; 88 (3): F185-F189. doi: 10.1136/fn.88.3.f185.

3. Pondaag W, Malessy MJA, Van Dijk JG, Thomeer RTW Natural history of obstetric brachial plexus palsy: a systematic review. Developmental Medicine \& Child Neurology. 2004; (46): 138-144. doi: 10.1111/j.1469-8749.2004.tb00463.x.

4. Ho E, Davidge K, Curtis C, Clarke H. Sensory outcome in children following microsurgery for brachial plexus birth injury. J Hand Surg Am. 2019; 44 (2): 159.e1-159.e8.

5. Ribeiro PR, Sparapani FV. Paralisia obstétrica do plexo braquial. Rev Bras Neurol Psiquiatr. $2014 ; 18$ (2): $148-155$.

6. Cunha MR, Dias AA, Brito JM, Cruz CS, Silva SK. Anatomical study of the brachial plexus in human fetuses and its relation with neonatal upper limb paralysis. einstein (São Paulo). 2020; 18: eAO5051. http://dx.doi.org/10.31744/ einstein_journal/2020AO5051.

7. Moore KL, Dalley AF, Agur AM. Anatomia orientada para a clínica. Rio de Janeiro: Editora Guanabara Koogan; 2018.

8. Abzug JM, Kozin SH. Evaluation and management of brachial plexus birth palsy. Orthop Clin North Am. 2014; 45 (2): 225-232. doi: 10.1016/j.ocl.2013.12.004.

9. Backe B, Magnussen EB, Johansen OJ, Sellaeg G, Russwurm H. Obstetric brachial plexus palsy: a birth injury not explained by the known risk factors. Acta Obstet Gynecol Scand. 2008; 87 (10): 1027-1032. doi:10.1080/00016340802415648.

10. Schmieg S, Nguyen JC, Pehnke M, Yum SW, Shah AS. Team approach: management of brachial plexus birth injury. JBJS Reviews. 2020; 8 (7): e19.00200. doi: 10.2106/JBJS.RVW.19.00200.

11. Woźniak J, Kędzia A, Dudek K. Variability of the trunks and divisions of the brachial plexus in human fetuses. Adv Clin Exp Med. 2013; 22 (3): 309-318.

12. Abdouni YA, Checoli GF, Nascimento VG, Costa AC, Chakkour I, Fucs PM. Relationship of age and type of obstetric brachial plexus paralysis in forearm pronosupination. Rev Bras Ortop. 2017; 52 (5): 596-600.

13. Van Dijk JG, Pondaag W, Malessy MJ. Obstetric lesions of the brachial plexus. Muscle Nerve. 2001; 24 (11): 1451-1461.

14. Benjamin K. Part 1. Injuries to the brachial plexus: mechanisms of injury and identification of risk factors. Adv Neonatal Care. 2005; 5 (4): 181-189. doi: 10.1016/j.adnc.2005.03.004.

15. Nath RK, Kumar N, Avila MB, et al. Risk factors at birth for permanent obstetric brachial plexus injury and associated osseous deformities. ISRN Pediatrics. 2012; 2012: 307039. doi: 10.5402/2012/307039.

16. Louden E, Marcotte M, Mehlman C, Lippert W, Huang B, Paulson A. Risk factors for brachial plexus birth injury. Children (Basel). 2018; 5 (4): 46. doi: 10.3390/children5040046.

17. Yarfi C, Elekusi C, Banson AN, Angmorterh SK, Kortei NK, Ofori EK. Prevalence and predisposing factors of brachial plexus birth palsy in a regional hospital in Ghana: a five year retrospective study. Pan Afr Med J. 2019; 32: 211. doi: 10.11604/pamj.2019.32.211.17914.

18. Gherman RB. Shoulder dystocia: an evidence-based evaluation of the obstetric nightmare. Clin Obstet Gynecol. 2002;4 5 (2): 345-362. doi:10.1097/00003081-200206000-00006.

19. Poggi SH, Stallings SP, Ghidini A, et al. Intrapartum risk factors for permanent brachial plexus injury. Am J Obstet Gynecol. 2003; 189: 725-729.

20. Gilbert A, Whitaker I. Obstetrical brachial plexus lesions. J Hand Surg Br. 1991; 16 (5): 489-491. doi: 10.1016/0266-7681(91)90100-3.

21. Michelow BJ, Clarke HM, Curtis CG, Zuker RM, Seifu Y, Andrews DF. The natural history of obstetrical brachial plexus palsy. Plast Reconstr Surg. 1994; 93 (4): 675-681. 
22. Clarke HM, Curtis CG. An approach to obstetrical brachial plexus injuries. Hand Clin. 1995; 11 (4): 563-581.

23. Mallet J. Paralysie obstétricale du plexus brachial. II. Thérapeutique. Traitement des séquelles. Primauté du traitement de l'épaule. Méthode d'expression des résultats [Obstetrical paralysis of the brachial plexus. II. Therapeutics. Treatment of sequelae. Priority for the treatment of the shoulder. Method for the expression of results]. Rev Chir Orthop Reparatrice Appar Mot. 1972; 58: 166-168.

24. Bae DS, Waters PM, Zurakowski D. Reliability of three classification systems measuring active motion in brachial plexus birth palsy. J Bone Joint Surg Am. 2003; 85 (9): 1733-1738. doi: 10.2106/00004623200309000-00012.

25. Coelho BB, Rocha LO, Guimaraes EM. Abordagem fisioterapêutica em criança com paralisia braquial obstétrica utilizando terapia de contenção e indução do movimento. C\&D Rev Eletrônica de Fainor. 2013; 6 (2): 127-149.

26. Amador EM. Parálisia obstétrica del plexo braquial. Revisión del estado actual de la enfermedad. Rev Fac Med. 2014; 62 (2): 255-263.

27. Curtis C, Stephens D, Clarke HM, Andrews D. The active movement scale: an evaluative tool for infants with obstetrical brachial plexus palsy. J Hand Surg Am. 2002; 27 (3): 470-478.

28. Abzug JM, Chafetz RS, Gaughan JP, Ashworth S, Kozin SH. Shoulder function after medial approach and derotational humeral osteotomy in patients with brachial plexus birth palsy. J Pediatr Orthop. 2010; 30 (5): 469-474.

29. Dawson A, Vasquez E, Garrett D Jr, Harris FS, EI Nihum IM, Dayawansa S, Huang JH, Singel S. Simultaneous Erb's and Klumpke's palsy: Case report. World J Clin Cases. 2015; 3 (12): 984-987. doi: 10.12998/wjcc.v3.i12.984.

30. Waters PM. Obstetric Brachial Plexus Injuries: Evaluation and Management. J Am Acad Orthop Surg. 1997; 5 (4): 205-214. doi: 10.5435/00124635-199707000-00004.

31. Bauer AS, Kalish LA, Adamczyk MJ, et al. Microsurgery for brachial plexus injury before versus after 6 months of age: results of the multicenter treatment and outcomes of brachial plexus injury (TOBI) study; treatment and outcomes of brachial plexus injury (TOBI) study group. J Bone Joint Surg Am. 2020; 102 (3): 194-204. doi: 10.2106/JBJS.18.01312.

32. Daly MC, Bauer AS, Lynch H, Bae DS, Waters PM. Treatment and outcomes of brachial plexus birth injury (TOBI) study group. Outcomes of late microsurgical nerve reconstruction for brachial plexus birth injury. J Hand Surg Am. 2020; 45 (6): 555.e1-555.e9. doi: 10.1016/j.jhsa.2019.10.036.

33. Garg R, Merrell GA, Hillstrom HJ, Wolfe SW. Comparison of nerve transfers and nerve grafting for traumatic upper plexus palsy: a systematic review and analysis. J Bone Joint Surg Am. 2011; 93 (9): 819-829. doi: 10.2106/JBJS.I.01602.

34. Coroneos CJ, Voineskos SH, Christakis MK, et al. Obstetrical brachial plexus injury (OBPI): Canada's national clinical practice guideline. BMJ Open. 2017; 7: e014141. doi: 10.1136/bmjopen-2016-014141.

35. Narakas AO. Obstetric brachial plexus injuries. In: Lamb DW (Ed.) The paralysed hand. Edinburgh, Churchill Livingstone, 1987: 116-135.

36. Al-Qattan MM, El-Sayed AA, Al-Zahrani AY, et al. Narakas classification of obstetric brachial plexus palsy revisited. J Hand Surg Eur Vol. 2009; 34 (6): 788-791. doi: 10.1177/1753193409348185.

37. Hosbay Z, Ozkan S, Tanriverdi M, Aydin A. Reliability and validity of the brachial plexus outcome measure in children with obstetric brachial plexus palsy. J Hand Ther. 2019; 32 (3): 382-387. doi:10.1016/j. jht.2017.10.006

38. Akbari A, Mayhew A, Al-Alawi MA, et al. Interventions to improve outpatient referrals from primary care to secondary care. Cochrane Database Syst Rev. 2008; (4): CD005471. 